\title{
Surface area regulation: underexplored yet crucial in cell motility
}

\section{Robert R. Kay, Paul Langridge, David Traynor and Oliver Hoeller}

In response to our review article (Changing directions in the study of chemotaxis. Nature Rev. Mol. Cell Biol. 9, 455-463 (2008)) ${ }^{1}$ we thank Maurice Hallett and co-workers (Chemotaxis and the cell surface-area problem. Nature Rev. Mol. Cell Biol. 23 July 2008 (doi:10.1038/nrm2419-c1) $)^{2}$ for their comments in which they emphasize the importance of the 'surface-area problem' in chemotaxis, as indeed they have done in the past for phagocytosis $^{3}$. The problem arises because motile cells often change shape in a way that increases their apparent surface area - they project pseudopodia or flatten out - yet they are possessed of a plasma membrane that is barely expandable 4 . To avoid rupturing their plasma membrane, motile cells therefore need some way of increasing their surface area on demand. In principle, this could be achieved either by unfolding surface wrinkles, as championed by Hallett and co-workers ${ }^{3}$, or by exocytosis of vesicles from within the cell, as we propose ${ }^{5}$. However these need not be stark alternatives, and evidence for both methods can be gleaned from cells with different physiologies; indeed both methods may be used by the same cell at different degrees of surface-area expansion.

Bladder epithelium expands and contracts cyclically as the bladder fills and empties. The epithelial surface is thought to initially expand by unfolding, but then new membrane is inserted into the plasma membrane and can be detected by increased capacitance (capacitance should give a readout proportional to surface area, whether the membrane is folded or not $)^{6,7}$. Lung epithelium may behave in a similar way to bladder epithelium ${ }^{8}$. Macrophages also increase their surface area as they engulf particles by phagocytosis: surface area increases as the cell extends a pseudopod around the particle, and then decreases when the phagosome is pinched off. Area increase can again be detected by capacitance and phagocytosis seems to depend on the exocytosis of membrane vesicles into the phagocytic cup ${ }^{9-11}$.

The surface area of an irregular, motile cell is less easily measured. Using reconstructions from optical sections we found that Dictyostelium discoideum cells can increase their surface area by $30 \%$ over a few minutes ${ }^{5}$. We did not see an unfolding of the membrane that might contribute to this, and total internal reflection fluorescence microscopy did not reveal folding of the lower membrane ${ }^{12}$. It is difficult to exclude a contribution from sub-microscopic folds, but we prefer to propose that these cells regulate their surface area mostly by changing the balance between exocytosis and endocytosis. This would also explain why an operational endocytic cycle is essential for motility in D. discoideum cells ${ }^{13}$.
Surface-area regulation is a little explored facet of cell motility, yet is likely to be crucial. Progress would be made if we could develop improved methods for measuring the surface area of motile cells and understand better their endocytic cycle and its possible regulation by membrane tension.

MRC Laboratory of Molecular Biology, Cambridge $\mathrm{CB} 2 \mathrm{OQH}$, UK

Correspondence to R.R.K.e-mail:rrk@mrc-Imb.ac.uk doi:10.1038/nrm2419-c2

1. Kay, R. R., Landridge, P., Traynor, D. \& Hoeller, O. Changing directions in the study of chemotaxis. Nature Rev. Mol. Cell Biol. 9, 455-463 (2008)

2. Hallett, M. B., von Ruhland, C. J. \& Dewitt, S. Chemotaxis and the cell surface-area problem. Nature Rev. Mol. Cell Biol. 23 July 2008 (doi:10.1038/ nrm2419-c1).

3. Hallett, M. B. \& Dewitt, S. Ironing out the wrinkles of neutrophil phagocytosis: membrane reservoirs for surface area expansion. Trends Cell Biol. 17, 209-214 (2007)

4. Mohandas, N. \& Evans, E. Mechanical properties of the red cell membrane in relation to molecular structure and genetic defects. Annu. Rev. Biophys. Biomol. Struct. 23, 787-818 (1994).

5. Traynor, D. \& Kay, R. R. Possible roles of the endocytic cycle in cell motility. J. Cell Sci. 120, 2318-2327 (2007).

6. Lewis, S. A. \& de Moura, J. Incorporation of cytoplasmic vesicles into apical membrane of mammalian bladder epithelium. Nature 297 685-688 (1982).

7. Truschel, S. T. et al. Stretch-regulated exocytosis/ endocytosis in bladder umbrella cells. Mol. Biol. Cell 13, 830-846 (2002)

8. Fisher, J. L., Levitan, I. \& Margulies, S. S. Plasma membrane surface increases with tonic stretch of alveolar epithelial cells. Am. J. Respir. Cell Mol. Biol. 31, 200-208 (2004).

9. Holevinsky, K. O. \& Nelson, D. J. Membrane capacitance changes associated with particle uptake during phagocytosis in macrophages. Biophys. J. 75, 2577-2586 (1998).

10. Hackam, D. J. et al. v-SNARE-dependent secretion is required for phagocytosis. Proc. Natl Acad. Sci. USA 95, 11691-11696 (1998)

11. Di, A. et al. Dynamin regulates focal exocytosis in phagocytosing macrophages. Mol. Biol. Cell 14, 2016-2028 (2003).

12. Gerisch, G. et al. Mobile actin clusters and traveling waves in cells recovering from actin depolymerization. Biophys. J. 87, 3493-3503 (2004).

13. Thompson, C. R L \& Bretscher, M. S. Cell polarity and locomotion, as well as endocytosis, depend on NSF. Development 129, 4185-4192 (2002). 